\title{
Lactose Sensitivity and Lactose Malabsorption: The 2 Faces of Lactose Intolerance
}

\author{
Michel Bouchoucha, ${ }^{1,2 *}$ Marinos Fysekidis, ${ }^{3}$ Pierre Rompteaux, ${ }^{2}$ Jean-Jacques Raynaud, ${ }^{2}$ Jean-Marc Sabate, ${ }^{2}$ \\ and Robert Benamouzig ${ }^{2}$
}

${ }^{1}$ Department of Physiology, Université René Descartes, Paris V, Paris, France; ${ }^{2}$ Department of Gastroenterology, Avicenne Hospital, Bobigny, France; and ${ }^{3}$ Nutrition and Diabetology, Hôpital Jean Verdier, Bondy, France

\section{Background/Aims}

Self-reported lactose intolerance (LI) is frequent in patients with functional bowel disorders (FBD) that could be interpreted as irritable bowel syndrome (IBS). The present study aims to characterize the responses of patients with FBD, without small intestinal bacterial overgrowth (SIBO), and LI, in terms of lactose malabsorption (LM) and lactose sensitivity (LS) according to psychological and clinical features.

\section{Methods}

One hundred and fifty-eight consecutive FBD outpatients with $\mathrm{LI}$, and no SIBO, were classified according to the Rome III questionnaire and filled Beck Depression Inventory, and State and Trait Anxiety questionnaires. They underwent a lactose tolerance test in which glycemia during 60 minutes and digestive symptoms for 3 hours were recorded.

\section{Results}

Abnormal lactose tolerance tests were found in 110 patients (70\%), 44 (28\%) with LM, $96(61 \%)$ with LS, and $30(19 \%)$ having both LM and LS. LM patients had a higher frequency of functional diarrhea $(P=0.040)$ and a lower frequency of dysphagia $(P=0.031)$. LS patients had a higher depression score $(P=0.007)$, higher frequency of globus $(P=0.042)$, irritable bowel syndrome (IBS) $(P=$ $0.027)$ and mixed IBS $(P=0.049)$, and lower frequency of abdominal pain $(P=0.040)$. LS was significantly associated with a higher depression score $(P=0.002)$, and a higher frequency of globus $(P=0.046)$.

\section{Conclusions}

Thirty percent of LI patients have normal lactose absorption and normal LS. In the other $70 \%$ of patients, LI could be associated with LM and/or LS.

(J Neurogastroenterol Motil 2021;27:257-264)

\section{Key Words}

Diarrhea; Irritable bowel syndrome; Lactose intolerance

\section{Received: April 28, 2020 Revised: June 25, 2020 Accepted: August 3, 2020}

(a) This is an Open Access article distributed under the terms of the Creative Commons Attribution Non-Commercial License (http://creativecommons. org/licenses/by-nc/4.0) which permits unrestricted non-commercial use, distribution, and reproduction in any medium, provided the original work is properly cited.

${ }^{*}$ Correspondence: Michel Bouchoucha, MD, PhD

Service de gastro-entérologie, CEFRED (Centre d'Exploration Fonctionnelle et de Rééducation Digestive), Hôpital Avicenne, 125, rue de Stalingrad, 93009 Bobigny Cedex, France

Tel: +33-148-957-432, Fax: +33-148-957-437, E-mail: michel.bouchoucha@aphp.fr 


\section{Introduction}

Lactose intolerance (LI) is a digestive discomfort following the ingestion of food containing lactose. This disaccharide is hydrolyzed by lactase, an enzyme present in the small intestinal brush border into glucose and galactose. ${ }^{1}$ Like other mammals, in humans, a physiological decrease in lactase activity is observed after weaning. This decline of the lactase activity is called primary adult-type hypolactasia and is evident from $8-12$ years onwards. ${ }^{2}$ This physiological phenomenon, occurring in a large proportion of individuals, is an autosomal recessive condition and results from the decline of lactase enzyme activity in the intestinal cells. ${ }^{3}$

In subjects with hypolactasia, lactose is not completely digested since the levels of lactase are insufficient in the lining of the duodenum and reaches, as other fermentable oligosaccharides, disaccharides, monosaccharides, and polyols (FODMAPs), the colon where it is fermented by bacteria. ${ }^{4}$ This process causes the production of hydrogen, carbon dioxide, and methane in the large intestine. It is used in an indirect test for lactose malabsorption (LM), the lactose breath test, considered commonly as the most reliable, noninvasive, and inexpensive technique.

The degradation of lactose in the colon creates a call for water in the small intestine, occasioning an acceleration of intestinal transit with diarrhea, gas, gut pain, bloating, and borborygmi. These symptoms may be present sometime after the ingestion of lactose when the undigested lactose reaches the colon and is degraded by colonic bacteria. They are similar to those of irritable bowel syndrome (IBS), ${ }^{6}$ and nearly two-thirds of IBS patients associate symptoms with eating a meal. ${ }^{7}$ Besides, small intestinal bacterial overgrowth (SIBO) has similar clinical features, and SIBO increases the likelihood of LI in patients with chronic diarrhea as a direct result of lactose fermentation in the small intestine. ${ }^{8}$ However, the most used diagnostic test for SIBO diagnosis is the breath test using glucose or lactulose as reactive. ${ }^{9}$ However, a positive lactose breath test could be associated as well as LM as SIBO.

The presence of digestive symptoms after a meal, including lactose, could induce these people to describe themselves to be lactose intolerant. In consequence, they exclude all dairy products from their diet on the assumption that lactose is present in significant amounts. In addition to the difficulty of following a restrictive diet in lactose, patients usually have low calcium intake, ${ }^{10}$ whereas many of the symptoms could be induced by the lipids that are present in the dairy products rather than lactose itself. ${ }^{11}$ On the other hand, although LI is a frequent complaint of patients with functional bowel disorders (FBD), these IBS-like disorders are often underrated. ${ }^{12}$

Many factors could influence the variability of clinical manifestations related to lactose ingestion: physiological like gastric emptying, small bowel transit time, water absorption capacity, patients' subjective sensitivity to pain, or nutritional like the fat content of foods with lactose. Also, SIBO could trigger a secondary LM, which in consequence can produce LI symptomatology. In addition to these various factors influencing the symptoms related to lactose ingestion, some patients with LI do not relate their symptoms with dairy products and make the diagnosis difficult. ${ }^{13}$ Moreover, non-intestinal symptoms such as headache, memory deterioration, musculoskeletal pain, heart rhythm disorders, and other allergic reactions are found in 20-80\% of patients with LI. ${ }^{14}$ We hypothesized that lactose may trigger symptoms of LI in some patients without LM. We named lactose sensitivity (LS) this disorder of unknown mechanism.

In the present study, we aim to characterize the responses of FBD patients with self-reported LI symptoms in terms of LM and LS according to psychological and clinical features.

\section{Materials and Methods}

\section{Experimental Procedure and Ethical Consideration}

The study was declared as a routine care evaluation in the French National Agency for drug safety (ANSM, Agence Nationale de Sécurité du Médicament et des produits de santé, decision number: 2019-A00171-56). For this type of study, formal consent is not required. All procedures contributing to this work comply with the ethical standards of the relevant national and institutional committees on human experimentation and with the Helsinki Declaration of 1975 , as revised in 2013.

\section{Study Design}

In this prospective study, patients with FBD that complain of LI were studied by the response to lactose ingestion in terms of LM and LS.

\section{Subjects}

Among the outpatients referred to the Gastroenterology Clinic of the Avicenne Hospital (Bobigny, France) between October 2012 and January 2019, we included 158 patients that reported LI. The presence of any organic diseases (ie, metabolic, endocrine, neurologic, and psychiatric etiologies), in particular, allergic disorders, celiac diseases, IBD, eosinophilic gastroenteritis were excluded by 
clinical, biological, and endoscopic examination. Also, all patients had a normal glucose hydrogen breath tests at most 4 weeks before inclusion to exclude a SIBO. All patients had a nutritional status assessment by a clinical dietitian. In adherence to nutritional guidelines, patients were provided sufficient calcium intake and adequate liposoluble vitamins.

\section{Clinical and Psychological Evaluation}

Patients in the gastroenterologist's office filled out a standard clinical Rome questionnaire. The interpretation was based on the Rome III criteria diagnosis for functional esophageal disorders, gastroduodenal disorders, bowel disorders, abdominal pain syndrome, and anorectal disorders, as previously described. ${ }^{15}$ Four 10-points (0-9) Likert scales for the cardinal signs of FBD (constipation, diarrhea, bloating, and abdominal pain) during the last week were also recorded. Psychometric evaluation was performed using the Beck Depression Inventory for depression levels and the State and Trait Anxiety Inventory for anxiety.

\section{Response to Lactose Ingestion}

Despite lactose activity assay by jejunal biopsy has been proposed as the "gold standard," this method is too invasive for outpatients' clinical evaluation, and cannot separate LM patients from LS patients. Also, despite its sensitivity, the lactose hydrogen breath test could not evaluate the LM severity because of its dichotomic response. Hence, to evaluate LS and LM, patients underwent a lactose tolerance test by ingestion of $25 \mathrm{~g}$ of lactose diluted in $250 \mathrm{~mL}$ of water after an overnight fast as previously described. ${ }^{16}$ For the study of LM, samples of capillary blood were taken to test glucose concentration at $0,15,30,45$, and 60 minutes, using a Precision XceedPro glycemia reader (Abbott, Rungis, France). For each time point, measurements were taken twice on the same sample, and the mean value was recorded. Patients were classified as LM, LS, LM and LS, and normal according to the results of lactose tolerance test. LM patients were characterized by a glycemia increase lower than $0.2 \mathrm{~g} / \mathrm{L}(1.2 \mathrm{mmol} / \mathrm{L})$ after the test. Patients were classified LS if they report digestive symptoms (nausea, bloating, diarrhea, borborygmi, and abdominal pain) during the 3 hours following the test. Patients were classified LM and LS if they report digestive symptoms and have glycemia increase lower than $0.2 \mathrm{~g} / \mathrm{L}$. They were classified as having symptoms.

\section{Statistical Methods}

IBM Statistical Package for Social Sciences version 20 (IBM Corp, Armonk, NY, USA) was used to carry out statistical analy- ses. For quantitative parameters, the results are expressed as mean $\pm \mathrm{SD}$, while for qualitative parameters, the results are expressed as number and percentage. Chi-square tests were used for the analysis of qualitative variables. One-way analysis of variance with Bonferroni correction was used to search for categorical differences among the groups.

Logistic regression with the backward selection that included LM and or LS as a dependent variable and systematically as independent variables, gender, body mass index, age, clinical disorders, and psychological profile was used for data analysis. Statistically significant variables $(P<0.05)$ remained in the adjusted model.

\section{Results}

\section{Patient Characteristics}

As shown in Table, the female gender was predominant in the studied population ( $73 \%$ female). The mean age of patients was $41.6 \pm 15.8$ years, and the mean body mass index was $24.7 \pm 6.0$ $\mathrm{kg} / \mathrm{m}^{2}$. None of these patients had abnormal nutritional status (calcium, vitamin D, etc). IBS was the most frequent disorder (64\%).

On the 158 patients complaining of LI, abnormal results in lactose tolerance tests were found in 110 patients (70\%), $44(28 \%)$ having LM, 96 (61\%) LS, 30 (19\%) having both LM and LS, and 14 having asymptomatic LM, while $30 \%$ of patients had neither LM nor LS (Figure). There was no association between these 2 disorders $(P=0.213)$.

\section{Characteristics of Lactose Malabsorption Patients}

As compared with patients with normal lactose absorption, the univariate analysis showed that LM patients have similar demographics, psychological and clinical characteristics except for functional diarrhea ( $7 \%$ vs $19 \% ; P=0.040$ ). Also, the rise of glucose blood after lactose ingestion was lower in patients with LM $(0.13 \pm$ 0.05 vs $0.44 \pm 0.22 \mathrm{~g} / \mathrm{L} ; P<0.001)$.

The logistic regression showed that LM was associated, not significantly, with male gender (34\% vs $24 \% ; P=0.060$; OR, 0.447 ; $95 \%$ CI, $0.193-1.036)$, IBS (70\% vs $61 \% ; P=0.051$; OR, 2.408 ; 95\% CI, 0.996-5.822), and significantly with lower prevalence of dysphagia ( $11 \%$ vs $19 \% ; P=0.031$; OR, $0.187 ; 95 \% \mathrm{CI}$, 0.041-0.858).

\section{Characteristics of Patients With Lactose Sensitivity}

Patients with LS are also characterized by a lower rise of blood glucose $(0.31 \pm 0.19$ vs $0.41 \pm 0.29 \mathrm{~g} / \mathrm{L} ; P=0.019)$. These 


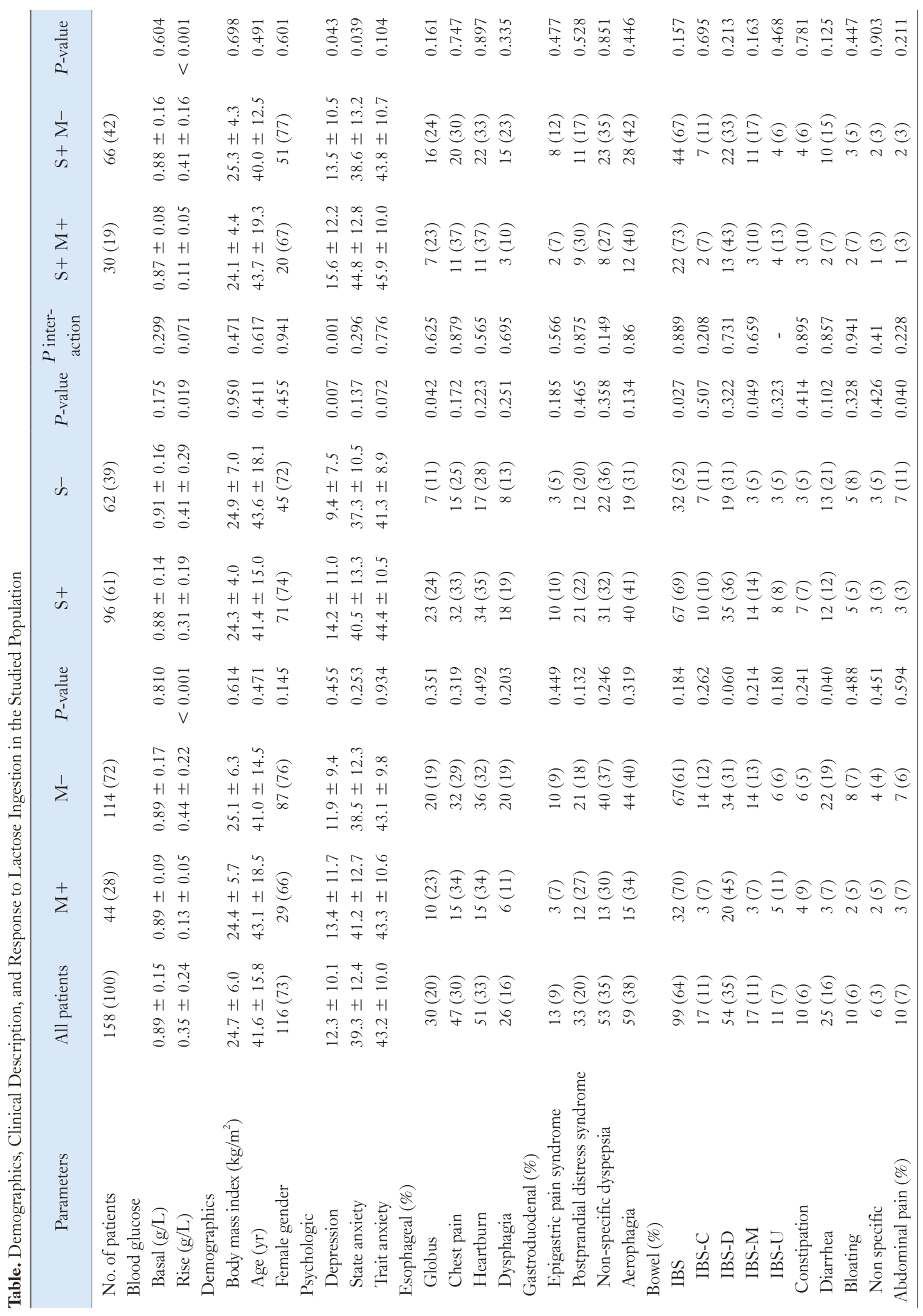




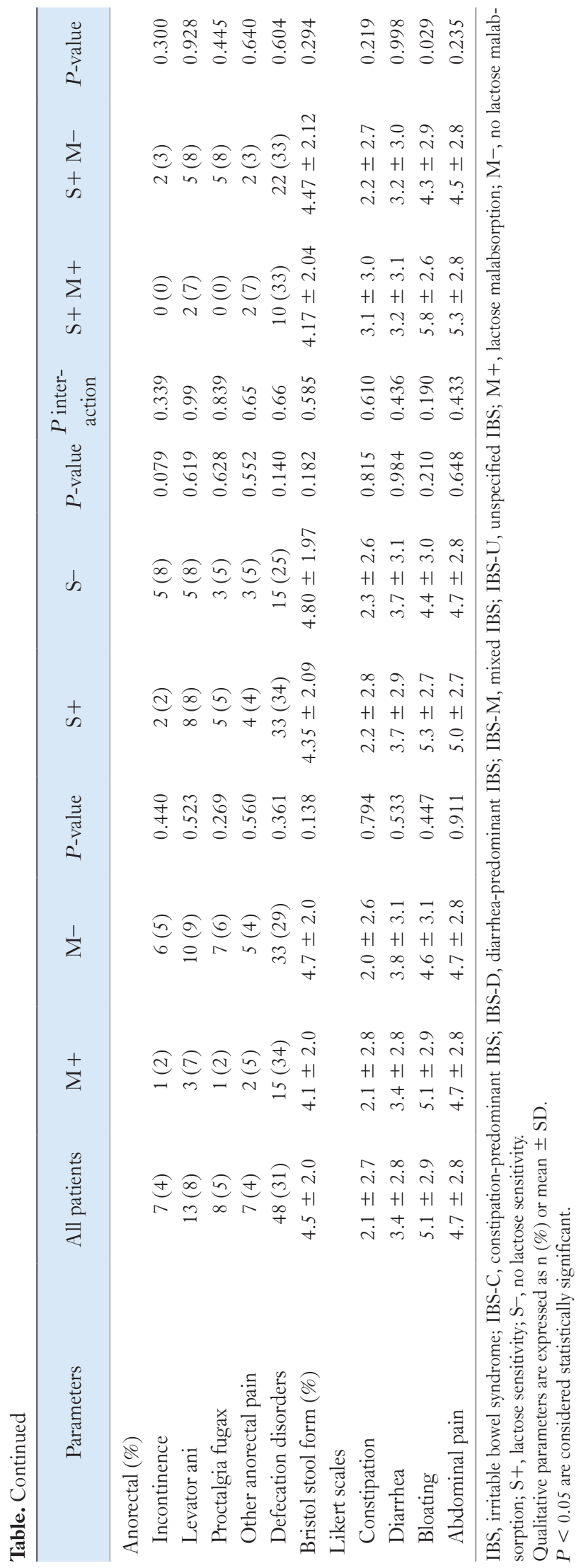

patients also have a higher depression score (14.2 \pm 11.0 vs $9.4 \pm$ $7.5 ; P=0.007)$, higher frequency of globus $(P=0.042)$, IBS $(P=0.027)$ and mixed IBS $(P=0.049)$, and lower frequency of abdominal pain $(P=0.040)$.

The logistic regression showed that patients with LS are associated with higher depression score $(P=0.002$; OR, 1.078 ; $95 \%$ CI, 1.028-1.131), higher frequency of globus ( $P=0.046$; OR, 2.956 ; 95\% CI, 1.018-8.586), lower frequency of dyspepsia ( $P=$ 0.030; OR, 0.377; 95\% CI, 0.156-0.911), and abdominal pain $(P=0.028$; OR, 0.169; 95\% CI, 0.035-.828).

\section{Characteristics of Patients With Functional Bowel Disorder According to Lactose Sensitivity and Lactose Malabsorption}

As compared with patients that have normal lactose absorption and no LS, the multinomial logistic regression shows that LS without $\mathrm{LM}$ patients has increased depression score $(P=0.006$; OR, $1.220 ; 95 \%$ CI, 1.059-1.406), whereas patients of the 2 other groups have no specific association.

\section{Discussion}

This study confirms the existence of a high frequency of digestive disorders induced by lactose ingestion $(61 \%)$ in patients that report FBD and LI. This study also shows the 2 faces of LI: LM and LS. However, despite that LM or LS was found in $70 \%$ of the participants, there was no association between these 2 disorders.

Both aspects of LI were previously reported in clinical and epidemiological studies. ${ }^{13}$ In humans, the physiopathology of digestive disorders associated with lactase deficiency is easy to understand. In the small intestine mucosa, lactase ( $\beta$-galactosidase), a disaccharidase present in the microvillar membrane of columnar epithelial cells hydrolyzed lactose into its component glucose and galactose. The lactase defect is responsible for the stagnation of undigested lactose in the small bowel that induces increased fluid into the caecum from the ileum by osmotic action. ${ }^{17}$ Lactases are also found in the microbiota, producing hydrogen, carbon dioxide, and shortchain organic acids. In North America and in Northern Europe, $80-90 \%$ of the adult population has high lactase activity, with no limit on milk or dairy consumption. ${ }^{18}$ However, we found a small group of asymptomatic LM patients ( $\mathrm{n}=14$ ) among our group of patients referred for LI. In these patients, we can speculate that the ingestion of $25 \mathrm{~g}$ of lactose was insufficient to induce symptoms. ${ }^{19}$

In contrast, the physiopathology of LS remains unknown. This disorder, also named "functional LI"1 was previously reported in 


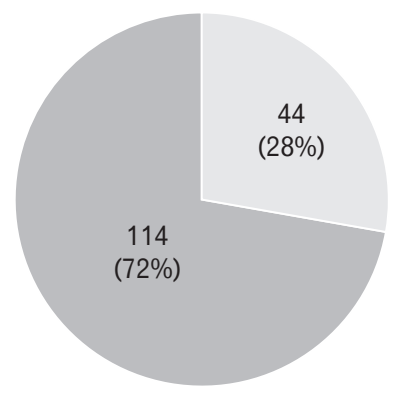

Lactose malabsorption No lactose malabsorption

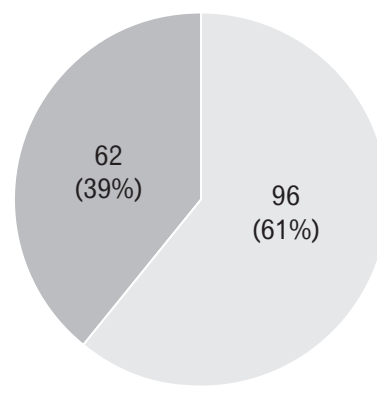

Symptoms No symptoms



Figure. Frequency of lactose malabsorption and lactose sensitivity in self-reported lactose intolerance patients without small intestinal bacterial overgrowth. Lactose sensitivity was more prevalent $(n=96[61 \%])$ than lactose malabsorption $(n=44[28 \%])$. Only 30 subjects $(19 \%)$ showed both disorders. $\mathrm{S}+\mathrm{M}+$, lactose sensitivity and lactose malabsorption; $\mathrm{S}-\mathrm{M}+$, lactose malabsorption without lactose sensitivity; $\mathrm{S}+\mathrm{M}-$, lactose sensitivity without lactose malabsorption; S- M-, no lactose malabsorption nor lactose sensitivity.

patients referred for investigation of digestive symptoms. The importance of a "double-blind, placebo-controlled food challenge" is considered as the "gold standard" for the diagnosis of food allergy. ${ }^{20}$ In a Chinese study, a cross-over comparison of lactose tolerance to 10,20 , and $40 \mathrm{~g}$ lactose showed that the proportion of patients with diarrhea-predominant IBS (IBS-D) reporting typical symptoms of LI was significantly higher at every dose compared to healthy subjects. ${ }^{6,21}$ Besides, IBS-D patients reported multiple and more severe symptoms than healthy subjects. However, in these studies, contrary to the present study, SIBO was not excluded before the lactose tolerance test, which is known to be frequent in IBS patients. ${ }^{22,23}$ A percentage of patients with IBS symptoms suffer from food hypersensitivity and are improved by a food-elimination diet. $^{24}$ Furthermore, in IBS patients, data from dietary elimination and rechallenge studies are poorly conclusive. ${ }^{24}$

In a Chinese group, patient complaints (abdominal pain, bloating, borborygmi, and symptom severity) after lactose ingestion were associated with visceral hypersensitivity, as measured by rectal barostat, and with high-levels of gas production on breath tests. ${ }^{25}$ The pathophysiological basis of LI in IBS-D patients was provided by a study showing increased counts of mast cells, increased intraepithelial lymphocytes, and increased enterochromaffin cells in the terminal ileum and the ascending part of the colon. Moreover, the release of pro-inflammatory cytokines after lactose ingestion was associated with hypervisceral sensitivity. ${ }^{6}$

IBS was the most prevalent FBD in which LM or LS was searched. It was previously found that despite the similar frequency of LM, IBS patients reported a higher frequency of LI than healthy subjects, ${ }^{26}$ avoid dairy products, ${ }^{21}$ and do not help in identifying lactose malabsorbers. ${ }^{27}$ Similarly, it was found that abnormal lactose breath tests in IBS subjects do not seem to reflect LM. ${ }^{28}$ These authors associated abnormal lactose breath tests with bacterial overgrowth, ${ }^{28}$ but in the present study, SIBO was excluded in all subjects before the LI study.

As in the present study, previous studies have hypothesized that LM was independent of IBS. ${ }^{29}$ However, the present study shows a higher IBS frequency, mainly mixed IBS, in patients with LS (Table). Similarly, a recent meta-analysis found that IBS patients report a higher frequency of LS, but not LM as compared with healthy controls. ${ }^{30}$ According to this point of view, LS could be interpreted as a marker of FODMAPS sensitivity. ${ }^{31}$ However, as shown in Table, LS was not only found in IBS patients but was found in all groups of patients with FBD.

Besides, patients with LS, but not patients with LM, have higher depression scores (Table) in agreement with earlier studies that have shown a higher prevalence of depression in German patients that reported LI as compared to other patients, ${ }^{14}$ and high depression score in a small group of female patients with $\mathrm{LM}^{32}$ or $\mathrm{LI}^{33}$ In contrast, a previous preliminary study showed that LM patients have a higher depression score. ${ }^{32}$ In the present study, State and Trait Anxiety were not different in LM patients as compared with normal absorbers, and in patients with LS as compared with patients without LS, contrarily to a previous Chinese study showing an increase of anxiety in IBS-D patients with LS. ${ }^{6}$

The main strength of this study is to show in a population of functional gastrointestinal disorders, without SIBO, fully explored (clinical and endoscopic examination, breath test), the self-report of LI, is not associated with LM or LS. Only $70 \%$ of these patients had LM, LS, or both. Among these patients, only a small percentage of these patients (19\%) having both symptomatic LM (simul- 
taneous presence of LM and LS). However, this study has some limits. The principal limit is the use of a non-double blinded test with a single lactose dose to study the sensitivity, although we know that the intensity of symptoms is dose-dependent. ${ }^{21}$

To conclude, the present study shows that $30 \%$ of patients that report LI have normal lactose absorption and normal LS. In the other $70 \%$ of patients, LI could be associated with LM (9\%), LS (42\%), or both LM and LS (19\%).

\section{Financial support: None.}

\section{Conflicts of interest: None.}

Author contributions: Michel Bouchoucha, Marinos Fysekidis, Pierre Rompteaux, Jean-Jacques Raynaud, Jean-Marc Sabate, and Robert Benamouzig contributed to the conception or design of the work; the acquisition, analysis, or interpretation of data of data for the work; drafting the article or revising it critically for important intellectual content; final approval of the version to be published; and agreement to be accountable for all aspects of the work in ensuring that questions related to the accuracy or integrity of any part of the work are appropriately investigated and resolved.

\section{References}

1. Misselwitz B, Pohl D, Frühauf H, Fried M, Vavricka SR, Fox M. Lactose malabsorption and intolerance: pathogenesis, diagnosis and treatment. United European Gastroenterol J 2014;1:151-159.

2. Schirru E, Corona V, Usai-Satta P, et al. Decline of lactase activity and c/t-13910 variant in Sardinian childhood. J Pediatr Gastroenterol Nutr 2007;45:503-506.

3. Vesa TH, Marteau P, Korpela R. Lactose intolerance. J Am Coll Nutr 2000;19(2 suppl):165S-175S.

4. Staudacher HM, Whelan K. The low FODMAP diet: recent advances in understanding its mechanisms and efficacy in IBS. Gut 2017;66:15171527.

5. Gasbarrini A, Corazza GR, Gasbarrini G, et al. Methodology and indications of H2-breath testing in gastrointestinal diseases: the Rome Consensus Conference. Aliment Pharmacol Ther 2009;29(suppl 1):1-49.

6. Yang J, Fox M, Cong Y, et al. Lactose intolerance in irritable bowel syndrome patients with diarrhoea: the roles of anxiety, activation of the innate mucosal immune system and visceral sensitivity. Aliment Pharmacol Ther 2014;39:302-311.

7. Schindler V, Giezendanner S, Bütikofer S, et al. Differentiation of functional gastrointestinal disorders from healthy volunteers by lactulose hydrogen breath test and test meal. J Gastroenterol Hepatol 2019;34:843851.

8. Zhao J, Fox M, Cong Y, et al. Lactose intolerance in patients with chronic functional diarrhoea: the role of small intestinal bacterial overgrowth. Ali- ment Pharmacol Ther 2010;31:892-900

9. Posserud I, Stotzer PO, Björnsson ES, Abrahamsson H, Simrén M. Small intestinal bacterial overgrowth in patients with irritable bowel syndrome. Gut 2007;56:802-808.

10. Carroccio A, Montalto G, Cavera G, Notarbatolo A. Lactose intolerance and self-reported milk intolerance: relationship with lactose maldigestion and nutrient intake. Lactase Deficiency Study Group. J Am Coll Nutr 1998;17:631-636.

11. Bouchoucha M, Devroede G, Raynaud JJ, Bon C, Bejou B, Benamouzig R. Is the colonic response to food different in IBS in contrast to simple constipation or diarrhea without abdominal pain? Dig Dis Sci 2011;56:2947-2956.

12. Hahn BA, Saunders WB, Maier WC. Differences between individuals with self-reported irritable bowel syndrome (IBS) and IBS-like symptoms. Dig Dis Sci 1997;42:2585-2590.

13. Ugidos-Rodríguez S, Matallana-González MC, Sánchez-Mata MC. Lactose malabsorption and intolerance: a review. Food Funct 2018;9:4056-4068.

14. Schiffner R, Kostev K, Gothe H. Do patients with lactose intolerance exhibit more frequent comorbidities than patients without lactose intolerance? An analysis of routine data from German medical practices. Ann Gastroenterol 2016;29:174-179.

15. Bouchoucha M, Devroede G, Mary F, Bon C, Bejou B, Benamouzig R. Both men and women with functional gastrointestinal disorders suffer from a high incidence of sexual dysfunction. Clin Res Hepatol Gastroenterol 2017;41:e93-e96.

16. Alexandre V, Davila AM, Bouchoucha M, et al. Agreement between indirect calorimetry and traditional tests of lactose malabsorption. Dig Liver Dis 2013;45:727-732.

17. Bond JH, Levitt MD. Quantitative measurement of lactose absorption. Gastroenterology 1976;70:1058-1062.

18. Storhaug CL, Fosse SK, Fadnes LT. Country, regional, and global estimates for lactose malabsorption in adults: a systematic review and metaanalysis. Lancet Gastroenterol Hepatol 2017;2:738-746.

19. Malik TF, Panuganti KK. Lactose intolerance. In: StarPearls [Internet]. Treasure Island (FL): StatPearls Publishing LLC 2020.

20. Asero R, Fernandez-Rivas M, Knulst AC, Bruijnzeel-Koomen CA. Double-blind, placebo-controlled food challenge in adults in everyday clinical practice: a reappraisal of their limitations and real indications. Curr Opin Allergy Clin Immunol 2009;9:379-385.

21. Yang J, Deng Y, Chu H, et al. Prevalence and presentation of lactose intolerance and effects on dairy product intake in healthy subjects and patients with irritable bowel syndrome. Clin Gastroenterol Hepatol 2013;11:262-268, e1.

22. Ghoshal UC, Kumar S, Mehrotra M, Lakshmi C, Misra A. Frequency of small intestinal bacterial overgrowth in patients with irritable bowel syndrome and chronic non-specific diarrhea. J Neurogastroenterol Motil 2010;16:40-46.

23. Shimura S, Ishimura N, Mikami H, et al. Small intestinal bacterial overgrowth in patients with refractory functional gastrointestinal disorders. J Neurogastroenterol Motil 2016;22:60-68.

24. Zar S, Kumar D, Benson MJ. Food hypersensitivity and irritable bowel 
syndrome. Aliment Pharmacol Ther 2001;15:439-449.

25. Zhu Y, Zheng X, Cong Y, et al. Bloating and distention in irritable bowel syndrome: the role of gas production and visceral sensation after lactose ingestion in a population with lactase deficiency. Am J Gastroenterol 2013;108:1516-1525.

26. Vesa TH, Seppo LM, Marteau PR, Sahi T, Korpela R. Role of irritable bowel syndrome in subjective lactose intolerance. Am J Clin Nutr 1998;67:710-715.

27. Vernia P, Marinaro V, Argnani F, Di Camillo M, Caprilli R. Self-reported milk intolerance in irritable bowel syndrome: what should we believe? Clin Nutr 2004;23:996-1000.

28. Pimentel M, Kong Y, Park S. Breath testing to evaluate lactose intolerance in irritable bowel syndrome correlates with lactulose testing and may not reflect true lactose malabsorption. Am J Gastroenterol 2003;98:27002704.
29. Vernia P, Di Camillo M, Marinaro V. Lactose malabsorption, irritable bowel syndrome and self-reported milk intolerance. Dig Liver Dis 2001;33:234-239.

30. Varjú P, Gede N, Szakács Z, et al. Lactose intolerance but not lactose maldigestion is more frequent in patients with irritable bowel syndrome than in healthy controls: a meta-analysis. Neurogastroenterol Motil 2018:e13527.

31. Lowe AW, Moseley RH. Role of dietary FODMAPs in IBS-related symptoms. Gastroenterology 2014;146:1-4.

32. Ledochowski M, Sperner-Unterweger B, Fuchs D. Lactose malabsorption is associated with early signs of mental depression in females: a preliminary report. Dig Dis Sci 1998;43:2513-2517.

33. Dainese R, Casellas F, Mariné-Barjoan E, et al. Perception of lactose intolerance in irritable bowel syndrome patients. Eur J Gastroenterol Hepatol 2014;26:1167-1175. 